\title{
Severe Hemorrhagic Shock Leads to a Delayed Fracture Healing and Decreased Bone Callus Strength in a Mouse Model
}

\author{
Katrin Bundkirchen MS, Christian Macke MD, Janin Reifenrath PhD, \\ Luisa Marilena Schäck PhD, Sandra Noack PhD, Borna Relja PhD, \\ Philipp Naber MS, Bastian Welke PhD, Michael Fehr VMD, \\ Christian Krettek MD, Claudia Neunaber PhD (D)
}

Received: 27 January 2017 / Accepted: 2 August 2017/Published online: 9 August 2017

(C) The Association of Bone and Joint Surgeons (B) 2017

\begin{abstract}
Background Multiple trauma is frequently associated with hemorrhagic shock and fractures of the extremities. Clinically, the rate of impaired fracture healing (delayed healing and nonunion) seems to be increased in patients with multiple injuries compared with patients with isolated fractures. As the underlying pathogenesis remains poorly understood, we aimed to analyze the biomechanical properties during fracture healing in a murine model.

Questions The aim of this study was to determine whether fracture healing after severe hemorrhagic shock results in (1) delayed bridging as determined by macroscopic and radiographic assessment, (2) altered conditions of callus
\end{abstract}

The institution of one or more of the authors (KB, CM, LMS, SN, CK, and $\mathrm{CN}$ ) has received, during the study period, funding from Deutsche Forschungsgemeinschaft (grant number NE 1932/1-1).

All ICMJE Conflict of Interest Forms for authors and Clinical Orthopaedics and Related Research ${ }^{\mathbb{B}}$ editors and board members are on file with the publication and can be viewed on request.

Clinical Orthopaedics and Related Research ${ }^{\mathbb{R}}$ neither advocates nor endorses the use of any treatment, drug, or device. Readers are encouraged to always seek additional information, including FDAapproval status, of any drug or device prior to clinical use.

Each author certifies that his or her institution approved the animal protocol for this investigation and that all investigations were conducted in conformity with ethical principles of research.

The work was performed in the Hannover Medical School, Hannover, Germany.

K. Bundkirchen, C. Macke, L. M. Schäck, S. Noack, C. Krettek,

C. Neunaber $(\square)$

Trauma Department, Hannover Medical School, Hannover,

Germany

e-mail: Neunaber.Claudia@mh-hannover.de

J. Reifenrath, M. Fehr

Small Animal Clinic, University of Veterinary Medicine,

Hannover, Germany components as determined by $\mu \mathrm{CT}$, and (3) decreased maximum bending moment measured by a three-pointbending test compared with ordinary fracture healing.

Methods Male C57BL/6NCrl mice were randomly assigned to five groups and four different times (five to 10 mice per group and time). Only the right femur from each mouse was used for analysis: the trauma hemorrhage (TH) group received a pressure-controlled hemorrhagic shock via catheter; the osteotomy ( $\mathrm{Fx}$ ) group underwent osteotomy and implantation of an external fixator on the right femur; the combined trauma (THFx) group received hemorrhagic shock and an external fixator with osteotomy; the sham group underwent implantation of a catheter and external fixator but had no blood loss or osteotomy, and the control group underwent no interventions. After 2, 3, 4, or 6 weeks, five to 10 animals of each group were sacrificed. Bones were analyzed macroscopically and via radiographs, $\mu \mathrm{CT}$, and three-point-bending test. Statistical significance was set at a probability less than 0.05 . Comparisons were performed using the Mann-Whitney U or the Kruskal-Wallis test.

Results In the Fx group, the osteotomy gap was stable and bridged after 2 weeks in contrast to some bones in the THFx group where stable bridging did not occur. No difference was observed between the groups. $\mu \mathrm{CT}$ analysis showed reduced density of bone including callus (THFx: $1.17 \mathrm{~g} /$ $\mathrm{cm}^{3}$; interquartile range [IQR], $0.04 \mathrm{~g} / \mathrm{cm}^{3} ; \mathrm{Fx}: 1.22 \mathrm{~g} / \mathrm{cm}^{3}$;

\section{J. Reifenrath}

Niedersächsisches Zentrum für Biomedizintechnik,

Implantatforschung und Entwicklung (NIFE), Lower Saxony Centre for Biomedical Engineering, Implant Research and Development, Department of Orthopedic Surgery, Hannover Medical School, Hannover, Germany 
IQR, $0.04 \mathrm{~g} / \mathrm{cm}^{3} ; \mathrm{p}=0.002$; difference of medians [DM], $-0.048 ; 95 \% \mathrm{CI},-0.073$ to -0.029$)$ and increased share of callus per volume of bone mass (\%) after 2 weeks in the THFx group compared with the Fx group (THFx: $44.16 \%$; IQR, 8.66\%; Fx: 36.73\%; IQR, 4.39\%; $\mathrm{p}=0.015$; DM, $7.634 ; 95 \%$ CI, 2.018-10.577). The three-point-bending test established a decreased maximum bending moment in the THFx group compared with the Fx group 2 weeks after surgery (THFx: $7.10 \mathrm{Nmm}$; IQR, $11.25 \mathrm{Nmm}$; Fx: 11.25 $\mathrm{Nmm}$; IQR, $5.70 \mathrm{Nmm}$; $=0.026$; DM, -5.043 ; 95\% CI, -10.867 to -0.74$)$. No differences were observed between the THFx and Fx groups after more than 2 weeks.

Conclusion In this in vivo mouse fracture model, we conclude that hemorrhagic shock retards fracture healing during the early phase of the facture healing process.

Clinical Relevance A severe hemorrhagic shock in patients could result in initial delayed fracture healing and needs special attention. We plan to conduct a prospective, observational clinical research study to analyze if delayed fracture healing occurs in patients after severe blood loss.

\section{Introduction}

Multiple trauma frequently is associated with hemorrhagic shock and fractures of the extremities [24]. Hemorrhagic shock is particularly common in patients with polytrauma and femoral shaft fractures [13]. In the clinical setting, the rate of impaired fracture healing (delayed healing and nonunion) may be increased in these patients compared with patients with isolated fractures [2, 5, 15]. Femur fractures may result in approximately $2000 \mathrm{~mL}$ of blood loss, tibia fractures approximately $1000 \mathrm{~mL}$, and humerus fractures approximately $800 \mathrm{~mL}$, which corresponds to hemorrhagic shock of Class II or III [18]. A trauma-induced hemorrhage of 750 to $1250 \mathrm{~mL}$ represents a $15 \%$ to $20 \%$ blood loss, whereas a hemorrhage of 1250 to $2000 \mathrm{~mL}$ is equivalent to $25 \%$ to $40 \%$ of the entire human blood volume [18]. However, children and older patients are more prone to serious complications after blood loss than young, healthy adults [18], therefore, very young and very old patients may be at particular risk.

Conflicting results have been reported in experimental studies investigating fracture healing after severe blood loss in animals. Wichmann et al. [28] and Lichte et al. [16] reported that trauma hemorrhage leads to increased

\section{B. Relja}

Department of Trauma-, Hand- and Reconstructive Surgery, University Hospital Frankfurt, Frankfurt, Germany

P. Naber, B. Welke

Laboratory for Biomechanics and Biomaterials, Department of Orthopedics, Hannover Medical School, Hannover, Germany osteocyte necrosis and delayed fracture healing. By contrast, Lucas et al. [17] and Bumann et al. [6] reported a positive effect of trauma hemorrhage on fracture healing, including an increased rate of mineralization and osteoblast number and better vascularization of the fracture zone.

Therefore, the aim of our study was to determine if fracture healing is affected by trauma hemorrhage during a 6-week period in a standardized mouse model. To answer this question we performed a pressure-controlled hemorrhagic shock during a 90-minute period followed by reperfusion with four times the shed blood volume, as this method has the advantage of continuous monitoring of the blood pressure [21] and good reproducibility [22]. The main difference between the studies mentioned above and our study design is attaching an external fixator to the femur followed by an osteotomy instead of a bending fracture. Our decision to use external fixation was for good reproducibility and standardization of the method, and also because of current scientific knowledge of the Damage Control Orthopedics concept, which is the standard treatment procedure for severely injured patients in Germany and in our trauma department [13]. The Damage Control Orthopedics concept postpones definitive surgical stabilization of the fracture in severely injured patients to a later time when the acute phase of systemic recovery is over and an external fixator is used to create temporary fracture stability [13]. By contrast, the Early Total Care concept stands for definitive fracture management during the early phase of multiple trauma, performing extended surgical procedures like implanting intramedullary nails [13]. Severely injured patients especially benefit from the Damage Control Orthopedics concept in contrast to the Early Total Care concept which more often leads to pulmonary complications, a higher inflammatory response, and increases the incidence of organ dysfunction in patients with multiple trauma [13]. To verify the underlying model, the biomechanical properties of fracture healing after 2, 3, 4, and 6 weeks by macroscopic, radiographic, and $\mu \mathrm{CT}$ analyses, and by biomechanical testing using a three-point bending assay were evaluated.

We asked if fracture healing after a severe hemorrhagic shock results in (1) delayed bridging as determined by macroscopic and radiographic assessments, (2) altered conditions of callus components as determined by $\mu \mathrm{CT}$, and (3) decreased maximum bending moment measured by a threepoint-bending test compared with ordinary fracture healing.

\section{Methods}

Animal Care

All animal experiments were performed in accordance with the German animal welfare legislation. Experiments were 
approved by the local institutional animal care and research advisory committee and permitted by the local government of Lower Saxony, Germany (Approval No. 33.14-4250204-14/1406). In this study, male C57BL/6NCrl mice, approximately 12 weeks old and initially weighing $26.38 \pm 1.21 \mathrm{~g}$, obtained from Charles River Laboratories (Sulzfeld, Germany) were used. Animals were kept under standardized temperature $\left(21^{\circ} \mathrm{C} \pm 2^{\circ} \mathrm{C}\right)$, relative humidity (50\%), and artificial light-dark cycles (14 hours light, 10 hours dark). They were fed ad libitum (Altromin 1324 TPF; Altromin $\mathrm{GmbH}$, Lage, Germany) with free access to water and were not fasted before the experiments. The health status of animals was monitored daily in a blinded manner.

\section{Group Distribution}

Animals were randomly assigned to one of five groups: Control, Sham, trauma hemorrhage (TH), osteotomy (Fx), and combined trauma (THFx) (five to 10 animals per group) (Table 1). The Control group underwent no interventions (healthy animals). Animals in the Sham group received implantation of a catheter and an external fixator but blood loss was not induced and no osteotomy was performed. The $\mathrm{TH}$ group received a pressure-controlled hemorrhagic shock via catheter with mean arterial blood pressure maintained at $35 \pm 5 \mathrm{~mm} \mathrm{Hg}$ for 90 minutes. Afterward, resuscitation was performed with Ringer's solution being administered at four times the shed blood volume, for 30 minutes as previously described [20]. The Fx group underwent an osteotomy after an external fixator was attached to the right femur. The THFx group received a hemorrhagic shock and an external fixator with osteotomy. The study design of these five groups including three "control" groups (Control, Sham, TH) was chosen to clarify that trauma hemorrhage (TH) or implantation of a catheter and an external fixator (Sham) alone had no effect on unbroken bones. Four of the five groups (Sham, $\mathrm{TH}, \mathrm{Fx}, \mathrm{THFx}$ ) were divided into four subgroups where mice were sacrificed 2, 3, 4, or 6 weeks after surgery. Animal number per subgroup was variable in the observed groups (Table 1). The reason for group number variability was justified by the European Commission Guideline 2010/63/ EU [9]; it is advised by law to reduce the number of animals wherever possible. For that reason the German animal welfare legislation determined that the Control group had to consist of five instead of 10 animals. Furthermore, before start of the study the German animal welfare legislation decided that if no differences were observed after 3 and 4 weeks between the Fx and THFx groups, the number of animals had to be reduced to six per group for 6 weeks. The same was true for the Sham and TH groups. If no differences were observable between those two groups after 3 and 4 weeks, the number of animals for the other times had to be
Table 1. Number of animals per group and time

\begin{tabular}{llllll}
\hline Group & $\begin{array}{l}\text { Total number of } \\
\text { animals }\end{array}$ & $\begin{array}{l}2 \\
\text { weeks }\end{array}$ & $\begin{array}{l}3 \\
\text { weeks }\end{array}$ & $\begin{array}{l}4 \\
\text { weeks }\end{array}$ & $\begin{array}{l}6 \\
\text { weeks }\end{array}$ \\
\hline Control & 5 & & & & \\
Sham & 32 & 6 & 10 & 10 & 6 \\
TH & 32 & 6 & 10 & 10 & 6 \\
Fx & 36 & 10 & 10 & 10 & 6 \\
THFx & 40 & 14 & 10 & 10 & 6 \\
\hline
\end{tabular}

TH - trauma hemorrhage group; Fx - osteotomy group, THFx combined trauma group consisting of $\mathrm{TH}$ and Fx.

reduced to six animals. Only the right femur from each mouse was used for analysis. The Control group consisted of 13-week-old mice (ordered at 12-weeks and sacrificed after 1 week acclimatization) that were randomly chosen from different litters during the study. As we required 10 stable bridged bones for biomechanical testing, the number of animals in the 2-week THFx group was increased from 10 to 14 . This was necessary as explantation of the femurs after 2 weeks led to four unstable bridged bones that were not usable for biomechanical testing.

Body weight, body temperature, and activity of the mice were measured in all groups before trauma and before sacrifice. The activity was determined with the score developed by Barkhausen et al. [3]. All surgical procedures were performed after anesthetizing the animals using isoflurane (Baxter Deutschland GmbH, Unterschleißheim, Germany). For anesthetic induction an isoflurane concentration of $3 \%$ was used which was reduced throughout the operation to $2 \%$ to $1.5 \%$. In the shock phase, the concentration was further reduced to less than $1.5 \%$ to relieve the blood circulation. During and after the surgical procedures mice were warmed using infrared warming lamps and heating pads. Before the operation, $5 \mathrm{mg} / \mathrm{kg}$ body weight carprofen (Rimadyl ${ }^{\circledR}$; Zoetis Deutschland GmbH, Berlin, Germany) and $1 \mathrm{mg} / \mathrm{kg}$ body weight butorphanol (Torbugesic ${ }^{\circledR}$; Zoetis Deutschland GmbH, Berlin, Germany) were injected subcutaneously. For postoperative analgesia, $0.8 \mathrm{mg} / \mathrm{mL}$ Novaminsulfon Lichtenstein $^{\circledR} 500 \mathrm{mg}$ (Zentiva Pharma GmbH, Frankfurt am Main, Germany) was added to the drinking water for the first 3 days after surgery. After the surgery, the animals were housed individually in cages to avoid aggressive behavior between adult male mice and littermates reopening the surgical wound. The activity and health status of the animals were monitored daily in a blinded manner.

Induction of Trauma Hemorrhage

Before initiating the hemorrhagic shock, the mouse was anesthetized with isoflurane and fixed in a supine position. 
The medial left hind leg was shaved, disinfected with Kodan $^{\circledR}$ (Schülke \& Mayr GmbH, Norderstedt, Germany), and the femoral artery was exposed by an incision through the skin. The femoral artery was cannulated using polyethylene tubing (Becton Dickinson and Company, Sparks, MD, USA), and the initial blood pressure was measured with a blood pressure measuring cell (FMI TBD-1222; Föhr Medical Instruments $\mathrm{GmbH}$, Seeheim, Germany) and a measurement amplifier (MIO-0501 DC; Föhr Medical Instruments GmbH). Animals were bled from a physiologic mean arterial blood pressure of approximately $100 \mathrm{~mm} \mathrm{Hg}$ via the catheter until a mean arterial blood pressure of $35 \pm 5 \mathrm{~mm} \mathrm{Hg}$ was reached. Blood pressure was monitored constantly during blood collection. The blood loss equaled $30 \%$ to $40 \%$ of the whole blood volume (average blood loss $700 \mu \mathrm{L}, 34 \%$ of the whole blood) $[12,19]$. After 90 minutes of shock, the animals were resuscitated with four times the shed blood volume using Ringer's solution (Berlin-Chemie AG, Berlin, Germany). We used Ringer's solution for reperfusion because this is comparable to the human situation in our clinic, where Ringer'sbased solutions are used for reperfusion in emergency vehicles. The catheter was removed and the incision was flushed with prilocaine hydrochloride (Xylonest ${ }^{\circledR} 1 \%$, AstraZeneca $\mathrm{GmbH}$, Wedel, Germany) to reduce postoperative pain. Then the vessels were ligated and the incision was closed with interrupted sutures.

\section{Implantation of the External Fixator and Osteotomy}

As it was shown that bone healing after saw osteotomy shows a very close resemblance to 'normal' fracture healing [8], we decided to perform a standardized osteotomy instead of a bending fracture. For stabilization of the osteotomy a standardized external fixator system was used (MouseExFix simple L 100\%; RISystem AG, Davos, Switzerland). The MouseExFix is made of polyetheretherketone, a high-strength medical grade polymer and the MouseExFix MountingPin $0.45 \mathrm{~mm}$ is made of medical grade titanium-aluminium-niobium alloy. To ensure consistent offset between the femur and the MouseExFix, all mounting pins had the same length and always interlocked in the MouseExFix in the same position. For implantation of the external fixator, the mouse was laid on the left side. The right thigh was shaved and disinfected with $\operatorname{Kodan}^{\circledR}$ (Schülke \& Mayr GmbH). A longitudinal skin incision was made along the femur from the hip to the knee and the fascia lata was opened. The vastus lateralis and biceps femoris muscles were split bluntly and the tensor fasciae latae was lifted to expose the full length of the femur. Four drill holes then were made with a $0.45-\mathrm{mm}$ drill bit (RISystem AG) and the external fixator with its four $0.45-\mathrm{mm}$ mounting pins (RISystem AG) was installed. Between the two middle pins the mouse received a standardized osteotomy at the diaphysis of the right femur using a $0.44-\mathrm{mm}$ Gigli wire saw (RISystem AG). Before closing the wound the lesion was flushed with prilocaine hydrochloride (Xylonest ${ }^{\circledR} 1 \%$ ). Afterward the fascia lata was closed with a continuous suture and the skin with interrupted sutures respectively.

\section{Survival Rate and Physiologic Response}

In total 164 animals underwent surgery, of which 19 mice were excluded $(11.6 \%)$. The animals were excluded from the study for the following reasons: 11 mice died during the trauma hemorrhage procedure $(6.7 \%)$; one animal had circumferential growth of the right kidney; and one animal in the Fx group was in very poor general health 1 day after the operation. Two bones showed signs of bone lysis and therefore disintegrated during pin removal. One bridged bone broke during preparation for the $\mu \mathrm{CT}$ analysis, as it was stuck in the vial, and one bone was not frozen again after $\mu \mathrm{CT}$ and therefore was removed from the study owing to autolysis. Two bones were excluded after 6 weeks because of abnormal diagnostic findings possibly caused by biomechanical influences of the external fixator on the bone or by chronic infection. Nevertheless, three of the 6week animals had to be sacrificed 5 days before the scheduled date because they reopened the skin in the area of the external fixator by biting; these three mice were analyzed as 6-week animals as planned.

\section{Harvesting Procedure}

During the time between surgery and sacrifice of the mice no changes in body weight were observed between the groups. The same is true for the body temperature before and after the surgery and before surgery compared with before sacrifice. One day postoperatively, all mice had an activity score of 5 or 6 [3] and there were no differences between groups.

For sacrifice, the animals were anesthetized with isoflurane and cervical dislocation was performed. The surgically treated femur was carefully explanted. The surrounding soft tissue was removed and radiographs were taken of the bones including the fixator. Afterward the external fixator was carefully removed with a square box wrench $(0.70 \mathrm{~mm}$; RISystem AG) to prevent bone damage. The bones were stored at $-20^{\circ} \mathrm{C}$.

\section{Radiographs}

The right femurs of mice that underwent an osteotomy were photographed (Nikon Digital Camera D100; Nikon 
GmbH, Düsseldorf, Germany), and radiographed (PRACTIX 160, Philips Medical Systems, Hamburg, Germany) at $48 \mathrm{kV}$ and $6.3 \mathrm{mAs}$. Bridging of the fracture gap was evaluated macroscopically and on the basis of the radiographs. The evaluation was performed by three independent observers (KB, CN, CM) in a blinded manner according to the radiographic scoring system of Goldberg et al. [10]: 0 points $=$ nonunion; 1 point $=$ possible union; 2 points $=$ radiographic union.

\section{$\mu \mathrm{CT}$}

The right femurs of the mice with an osteotomy were examined using $\mu \mathrm{CT}$ (XTremeCT: Scanco Medical Zurich, Switzerland) to measure bone volume after 3,4 , and 6 weeks with the following parameters: slice thickness/isotropic $\quad$ voxels $=41 \quad \mu \mathrm{m} ; \quad$ voltage $=60 \quad \mathrm{kV}$; amperage $=900 \mu \mathrm{A}$; and integration time $=700 \mathrm{~ms}$. Data were analyzed using $\mu \mathrm{CT}$ software Version 6.0 (Scanco Medical). A specific threshold of grey values $(>95)$ was determined and used for all evaluations to calculate bone volume $\left(\mathrm{mm}^{3}\right)$ in the center between the two middle pins $(1.23 \mathrm{~mm})$ at the osteotomy position.

Because the $\mu \mathrm{CT}$ scanner was changed during the study procedure, the right femurs of the 2-week mice were scanned using the QuantumFX low-dose in vivo $\mu \mathrm{CT}$ (PerkinElmer, Waltham, MA, USA) to measure the altered conditions of callus components with the following parameters: slice thickness/isotropic voxels $=40 \times 40 \times$ $40 \mu^{3}$, tube voltage $=90 \mathrm{kVp}$, tube current $=200 \mu \mathrm{A}$, $\mathrm{FOV}=20 \times 20 \mathrm{~mm}^{2}$, and total scanning time $=2 \mathrm{~min}-$ utes. Two right legs of different mice were always scanned together with a custom-made bone mineral density phantom (QRM Quality Assurance in Radiology and Medicine $\mathrm{GmbH}$, Moehrendorf, Germany) with five inclusions of hydroxyapatite $\left(0.2-1.0 \mathrm{~g} / \mathrm{cm}^{3}\right)$. A region of interest was placed at the fracture position $\left(4 \times 4 \times 1.2 \mathrm{~mm}^{3}\right)$. As the callus always had a lower mineral content than the cortical bone, a lower brightness for the callus was achieved in the acquired CT data sets and therefore the callus was isolated from the cortical bone using threshold-based segmentation (Fig. 1). The bone density $\left(\mathrm{g} / \mathrm{cm}^{3}\right)$ and respective volume $\left(\mathrm{mm}^{3}\right)$ were measured for the bone including the callus, the bone mass without the callus, and the cortex only. Moreover the share of callus per volume of bone mass (\%) was calculated using the following formula: ((Volume of the bone including the callus -Volume of the bone mass without callus)/Volume of the bone including callus)*100.

\section{Three-point Bending Test}

The three-point-bending test was performed 2, 3, 4, and 6 weeks after the operation using the right femurs of all groups. Before the three-point-bending test the bones were

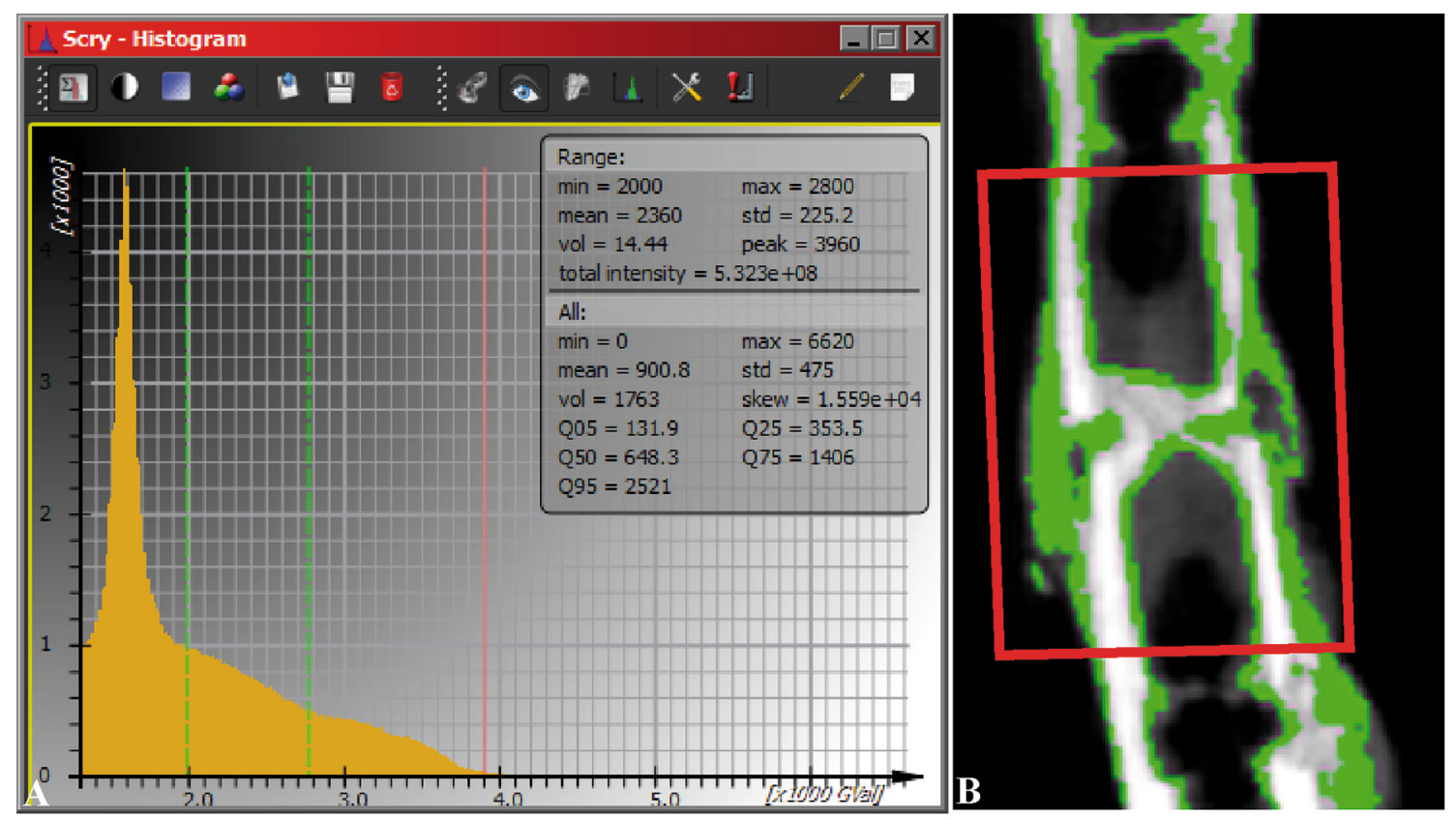

Fig. 1A-B The method of callus determination is shown. (A) The area between the two green lines in the histogram was assigned to callus. (B) The assigned callus is shown in green in the longitudinal bone section. The region of interest (red rectangle in illustration B) was placed at the fracture position $\left(4 \times 4 \times 1.2 \mathrm{~mm}^{3}\right)$ to calculate the callus. 
thawed at room temperature for 1 hour. Afterward, the bones were transferred to Ringer's solution to prevent dehydration before testing, however, the bones were not kept hydrated during testing. Before the actual test, the diameters of all harvested specimens at the mid-diaphysis were measured with an LDM 110 laser (Takikawa Engineering, Tokyo, Japan). The three-point bending test was performed using a materials testing machine (MTS Mini Bionix ${ }^{\circledR}$ I, Model 858; MTS Systems Corporation, Eden Prairie, MN, USA) with a custom-made three-point bending device. The three bearings of the loading supports were rounded to avoid damage to the samples. All bearings had a radius of $1.25 \mathrm{~mm}$ and the span length of the two lower bearings was $9.5 \mathrm{~mm}$. Each bone was positioned with the posterior surface downward, centered on the lower support. The upper support applied force vertically to the bone. The testing conditions provided no preload and were displacement-controlled at a constant velocity of the upper support of $3 \mathrm{~mm}$ per minute [1]. Deflection of the mid-diaphysis was measured by the linear displacement transducer of the actuator from the materials testing machine. The termination criterion of the experiment was defined as $3 \mathrm{~mm}$ deflection of the middiaphysis. At this point, all specimens had undergone complete structural failure. The maximum bending moment $(\mathrm{Nmm})$ and the stiffness $(\mathrm{N} / \mathrm{mm})$ were determined from the load-deformation curve. The stiffness $(\mathrm{N} /$ $\mathrm{mm}$ ) was taken in the linear part of the curve between $30 \%$ and $60 \%$ of the maximum applied load.

\section{Statistical Analysis}

Sample size calculation was determined before start of the study using G*Power Version 3.1.9.2 (University of Kiel, Germany). The following parameters were set in this program:
Comparison of Group 1 'Control' versus Group 2 'Fx' resulted in an effect size of 3.8. The calculated sample size for those two groups was three animals per group. Comparison of Group 2 'Fx' versus Group 3 'THFx' resulted in an effect size of 1.4. The calculated sample size for those two groups was eight animals per group. Therefore, a group size of eight animals per group and time was planned with two additional animals per group, in case an animal or a femur had to be excluded for some reason.

Statistical analysis was performed using SPSS Statistics Version 23.0 (IBM Corp, Armonk, NY, USA). All data were nonparametric. Therefore, comparisons between the THFx and Fx groups at the same time (Fx versus THFx after 2, 3, 4 or 6 weeks) were performed using the Mann-Whitney U test and comparisons between the other groups and at different times (Control versus Sham versus TH versus Fx versus THFx after 2 versus 3 versus 4 versus 6 weeks) with the Kruskal-Wallis test. Primary outcomes of the study were share of callus per volume of bone mass (\%) analyzed by $\mu \mathrm{CT}$ at 2 weeks and maximum bending moment measured by three-point bending assay between the Fx and THFx groups. The secondary outcomes were macroscopic and radiographic evaluations, bone volume after 3, 4, and 6 weeks, bone density including callus at 2 weeks measured by $\mu \mathrm{CT}$, and stiffness for the three-point bending assay. Results are expressed as median and interquartile range (eg, 2.9905; [IQR], 4.74). Results were considered statistically significant at a probability of 0.05 or less. For calculation of the difference of medians (DM), the Hodges-Lehmann estimate with a 95\% CI was used.

\section{Results}

Macroscopic and Radiographic Evaluations

Macroscopic evaluation of the bones 2 weeks after surgery (Fig. 2) revealed no difference, with the numbers available,

Type: “A priori: Compute required sample size - given $\alpha$, power and effect size"
$\begin{array}{ll}\text { Input parameters: } & \text { Two; } \\ \text { Tails: } & 0.05 \\ \alpha \text { err prob: } & 0.80\end{array}$

The effect size was determined by maximum bending moment after 2 weeks. The maximum bending values for the Control and Fx groups were known from preliminary experiments. Values for THFx group were predicted $(40 \%$ of Fx).

Group 1: Control (healthy animals) $\rightarrow 33 \pm 5 \mathrm{Nmm}$; Group 2: Fx $\rightarrow 14 \pm 5 \mathrm{Nmm}$;

Group 3: THFx $\rightarrow 6 \pm 5 \mathrm{Nmm}$. in the THFx group compared with the Fx group, with 0.92 points (IQR, 1.42 points) and 1.25 points (IQR, 0.54 points), respectively, regarding the osteotomy gap: the osteotomy gap of the Fx group was bridged in $100 \%$ of the mice (10 of 10), while just $72 \%$ of the osteotomy gaps of the THFx group were bridged (10 of 14) (DM, -0.333 ; $95 \% \mathrm{CI},-1$ to $0.167 ; \mathrm{p}=0.154)$. After 3 weeks and later we found no differences between the Fx and THFx groups, with the numbers available. 
Radiographic evaluation (Fig. 3) showed similar results as the macroscopic examination. After 2 weeks the osteotomy gap of the Fx group, with 1.17 points (IQR, 0.38 points), showed possible union, whereas the THFx group showed no radiologic signs of bridging callus in $35 \%$ of the mice (five of 14) with 0.67 points (IQR, 1.33 points) (Fig. 4). However, there was no difference between the groups, with the numbers available (DM, $-0.5 ; 95 \% \mathrm{CI},-1$ to $0 ; \mathrm{p}=0.138$ ). For all later times, no differences were observed between the Fx and THFx groups.

\section{$\mu \mathrm{CT}$ Evaluation}

Analysis of the 2-week animals by $\mu \mathrm{CT}$ showed decreased density of the bone including the callus in the THFx group compared with the Fx group, with $1.17 \mathrm{~g} / \mathrm{cm}^{3}$ (IQR, $0.04 \mathrm{~g} /$ $\mathrm{cm}^{3}$ ) and $1.22 \mathrm{~g} / \mathrm{cm}^{3}$ (IQR, $0.04 \mathrm{~g} / \mathrm{cm}^{3}$ ), respectively (DM, $-0.048 ; 95 \%$ CI, -0.073 to $-0.029 ; p=0.002$ ) (Fig. 5A). We also measured a higher share of callus per volume of bone mass (\%) in the THFx group compared with the Fx group, with $44.16 \%$ (IQR, 8.66\%) and 36.73\% (IQR,
4.39\%) respectively, after osteotomy (DM, 7.634; 95\% CI, 2.018-10.577; $\mathrm{p}=0.015$ ) (Fig. 5B).

No differences were found for volume of bone including the callus, volume of bone mass without the callus, volume of the cortex only, density of bone mass without the callus, and density of the cortex only. Again, at later times no differences in bone volume between the Fx and THFx groups were detectable by $\mu \mathrm{CT}$.

\section{Biomechanical Testing}

No difference was found in maximum bending moment between the Control, Sham, and TH groups at any time (Fig. 6A). In contrast, the THFx group showed a lower maximum bending moment than the Fx group, with 7.10 $\mathrm{Nmm}$ (IQR, $11.25 \mathrm{Nmm}$ ) and $11.25 \mathrm{Nmm}$ (IQR, 5.70 $\mathrm{Nmm}$ ), respectively 2 weeks after surgery (DM, -5.043 ; $95 \% \mathrm{CI},-10.867$ to $-0.74 ; \mathrm{p}=0.026$ ) (Fig. 6A). Moreover, the THFx and Fx groups showed a lower maximum bending moment than either the Sham group, with 34.26 Nmm (IQR, 8.13 Nmm) (DM, -28.727 [95\% CI, -35.162
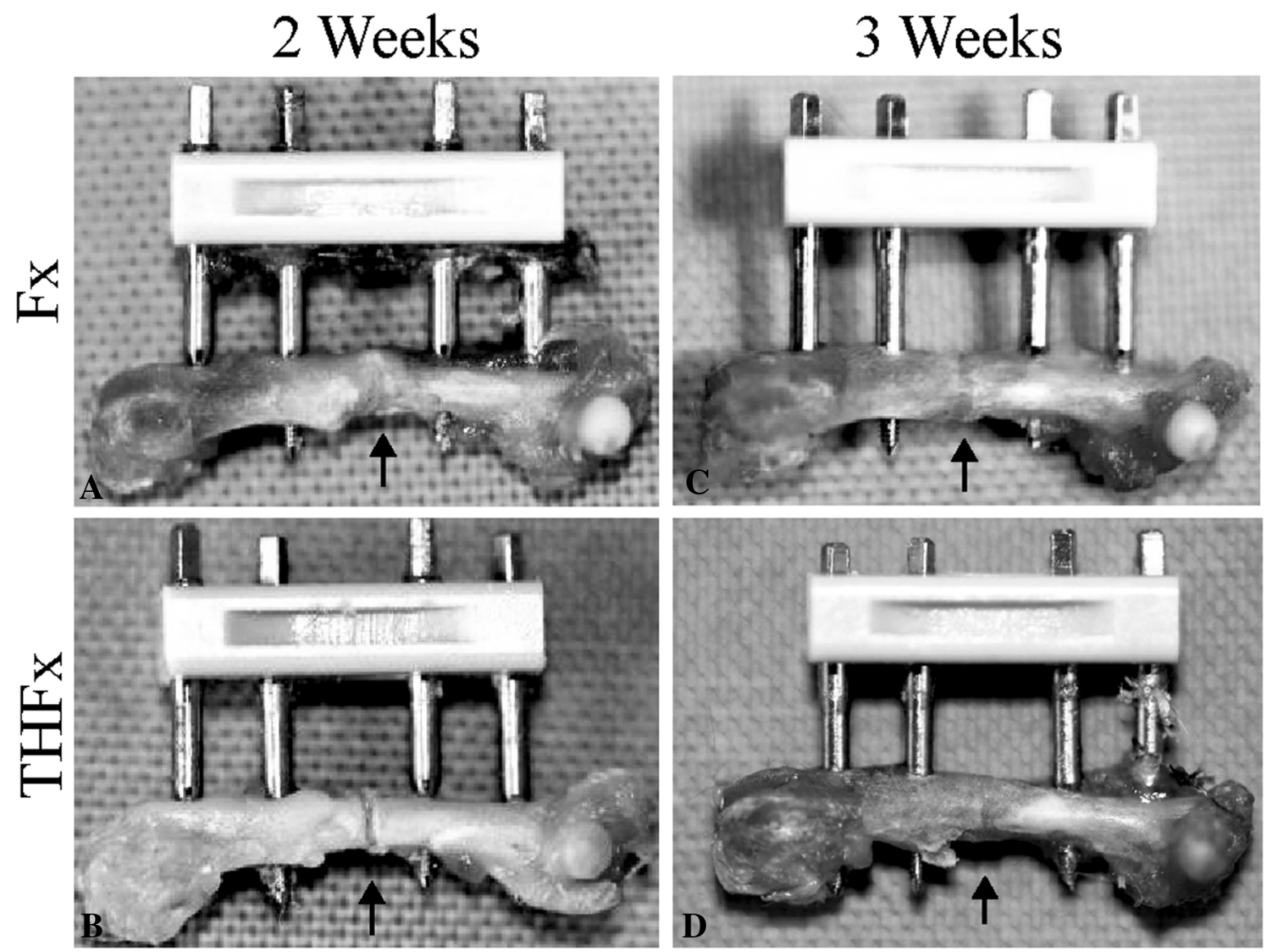

Fig. 2A-D (A) After 2 weeks, macroscopic bridging of the osteotomy gap for all animals in the Fx group was seen. (B) In the THFx group no bridging in $28 \%$ of the animals (four of 14) was determined. After 3 weeks the osteotomy gap is bridged in the (C) Fx and (D) THFx groups. The arrows point at the initial area of the fracture gap. 
Fig. 3A-D (A) Two weeks after surgery, radiographic bridging of the osteotomy gap in the Fx group was seen. (B) In the THFx group bridging cannot be seen in $35 \%$ of the cases (five of 14) after 2 weeks. After 3 weeks the osteotomy gap is bridged in the (C) Fx and (D) THFx groups.
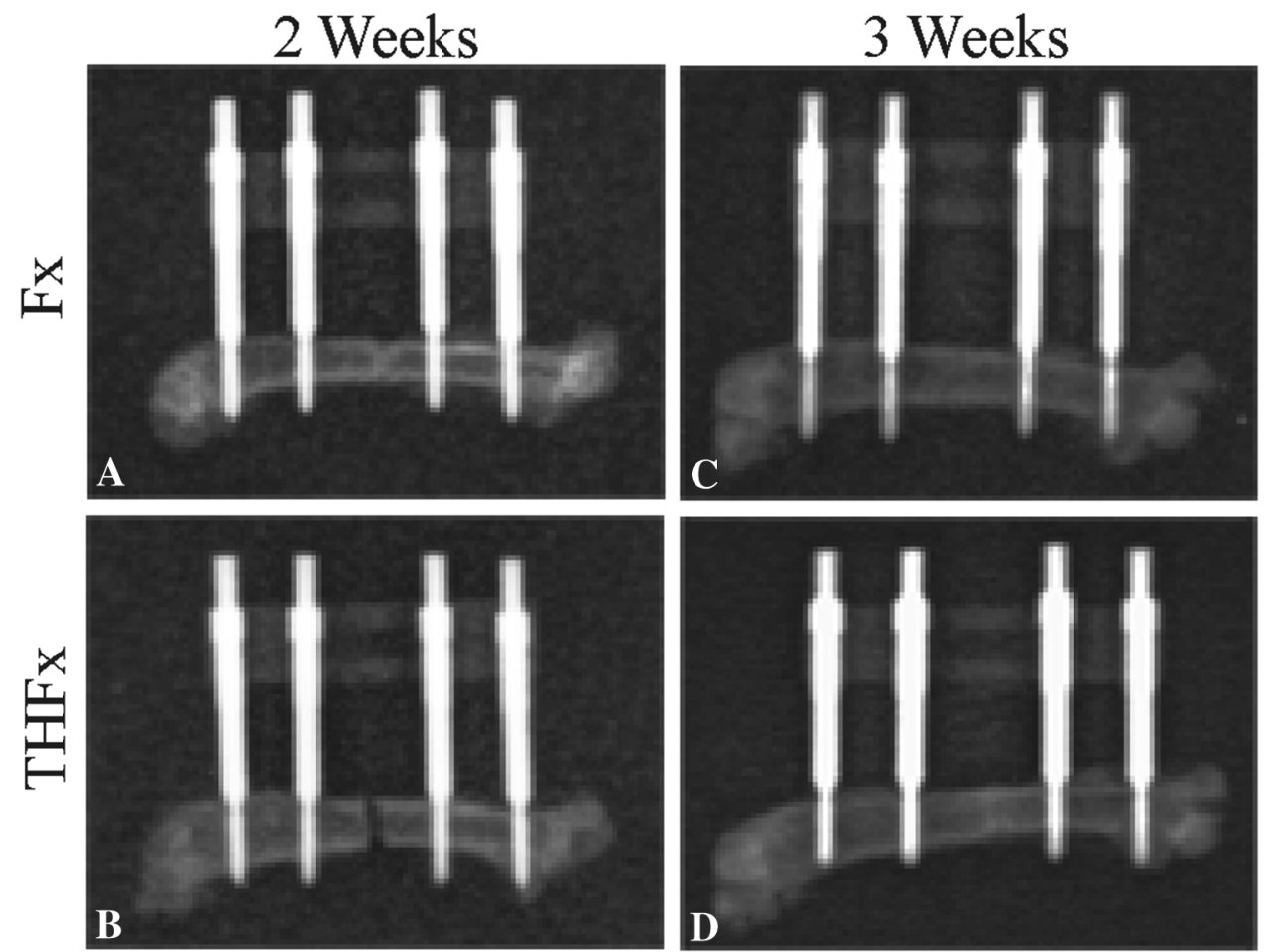

to -22.265 ] $\mathrm{p}=0.001$, versus the THFx group: DM, -22.647 [95\% CI, -28.924 to -17.562 ] $\mathrm{p}=0.015$, versus the Fx group) or the TH group with $34.77 \mathrm{Nmm}$ (IQR, 4.19 $\mathrm{Nmm}$ ) (DM, -27.969 [95\% CI, -32.42 to -23.186$]$ $\mathrm{p}=0.001$,versus the THFx group: DM, -23.166 [95\% CI, -26.84 to -17.372 ] $p=0.011$,versus the $F x$ group) (Fig. 6A). For the femurs explanted 3 weeks after surgery biomechanical testing revealed a difference in maximum bending moment between the Fx group with $18.68 \mathrm{Nmm}$ (IQR, 8.64 Nmm) and the Sham group with $36.56 \mathrm{~N}$ (IQR, $7.56 \mathrm{Nmm}$ ) (DM, -17.561 ; $95 \% \mathrm{CI},-22.606$ to -13.094 ; $\mathrm{p}=0.004)$, and between the THFx group with $21.42 \mathrm{Nmm}$ (IQR, 10.14 Nmm) and the Sham group (DM, -16.264; $95 \% \mathrm{CI},-22.507$ to $-12.032 ; \mathrm{p}=0.007$ ) (Fig. 6A). However, the difference between the Fx and THFx groups regarding the maximum bending moment that was present 2 weeks after surgery was not observable at 3 weeks (Fig. 6A). At the later times (4 and 6 weeks), no differences between any of the analyzed groups were observed by biomechanical testing (Fig. 6A).

With regard to stiffness, there was a difference between the THFx group with $9.05 \mathrm{~N} / \mathrm{mm}$ (IQR, $25.67 \mathrm{~N} / \mathrm{mm}$ ) compared with the TH group with $81.56 \mathrm{~N} / \mathrm{mm}$ (IQR, $25.29 \mathrm{~N} / \mathrm{mm}) 2$ weeks after surgery (DM, $-22.283 ; 95 \%$ $\mathrm{CI},-35.876$ to $-6.475 ; \mathrm{p}=0.002$ ) (Fig. 6B). No differences were observable between the Fx and THFx (Fig. 6B) groups after 3, 4, and 6 weeks nor between the other groups for all times.

\section{Discussion}

Impaired fracture healing, such as delayed healing or nonunion, frequently is observed in the clinical setting in patients with multiple injuries or after severe blood loss [5, 13]. Known reasons for delayed fracture healing or nonunion include a difficult fracture pattern or location, decreased soft tissue perfusion, and the presence of an infection [11]. Furthermore individual risk factors such as advanced age, smoking, or medical comorbidities are known to affect fracture healing [11]. In contrast, the effect of hemorrhagic shock on fracture healing in experimental models is still debated as several experimental studies have led to conflicting results $[6,10,17,28]$. In an attempt to clarify the effect of hemorrhagic shock on fracture healing we successfully established a standardized mouse model consisting of hemorrhagic shock, external fixator, and osteotomy in this study, and analyzed the macroscopic, radiographic, and biomechanical properties of the fracture-healing process after trauma hemorrhage during a 6-week course. We found: (1) reduced density of bone including callus and an increased share of callus per volume of bone mass (\%) analyzed by $\mu \mathrm{CT}$; and (2) decreased maximum bending moment analyzed by three-point bending test, respectively in the THFx group compared with the Fx group 2 weeks after surgery. No differences were observed between these two groups after more than 2 weeks. As fracture healing in mice is completed after 3 weeks [26], whereas fracture healing in humans lasts 

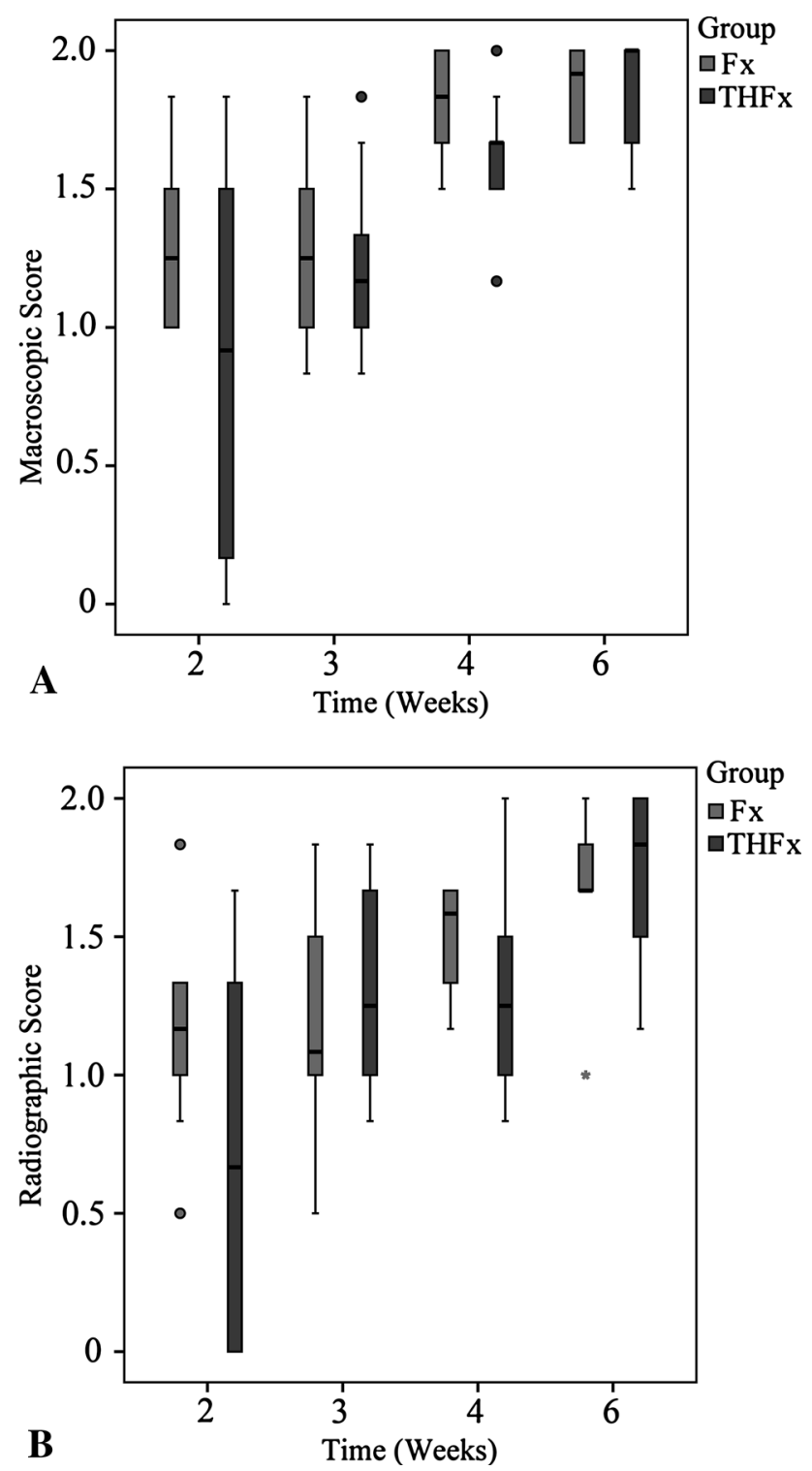

Fig. 4A-B There were no differences in the (A) macroscopic and (B) radiographic scores between the analyzed groups after 2,3 , 4, or 6 weeks.
12 to 16 weeks [23], a delayed fracture healing of 1 week in THFx group of mice could suggest a serious effect on human fracture healing. If we assume that fracture healing is four times faster in mice than in humans, a delay of 1 week in mice could suggest a delay in fracture healing of 4 weeks in humans. Therefore, these findings may be clinically relevant.

Some limitations of our study have to be considered. The model we chose for our study is a small animal model. This does not fully mimic the clinical situation in humans as young, male, healthy mice obviously cannot reflect the diversity of human patients of all ages, sexes, and genetic differences, illnesses, or habits like smoking or alcohol abuse. However, the mouse as a trauma model has the highest gene homology compared with humans and therefore is qualified for the analysis of fundamental regulatory mechanisms [14]. Apart from that, we used only male mice to avoid the influence of hormonal fluctuation on bone metabolism and inflammatory response after trauma hemorrhage which occurs in female mice [4, 7, 25]. Furthermore, we performed an osteotomy instead of a bending fracture to analyze fracture healing, as it was shown by Dumont et al. [8] that bone healing after saw osteotomy shows a close resemblance to 'normal' fracture healing. In addition, the increase of the density of the bone including the callus in the THFx group in contrast to the Fx group after 2 weeks was only $4 \%$. Because of this small difference it is unlikely that this result would show an effect in the clinical setting. Furthermore, the available voxel size of $40 \times 40 \times 40 \mu^{3}$ of the $\mu \mathrm{CT}$ analyses was insufficient to resolve the trabecular bone of mice, as trabecular bone typically has a mean trabecular distance of approximately $10 \mu \mathrm{m}$. Therefore, characterization of bone geometry and structure could not be performed. Apart from that, another possible limitation is that specimens were just initially hydrated at the beginning of the three-pointbending testing but not during testing. Even so, relative comparisons between groups should be reasonable because all bones were tested under the same conditions.
Fig. 5A-B (A) A decrease in bone density including callus and (B) an increase in the share of callus per volume of bone mass were observed between the Fx and THFx groups after 2 weeks. *p $\leq 0.05$.
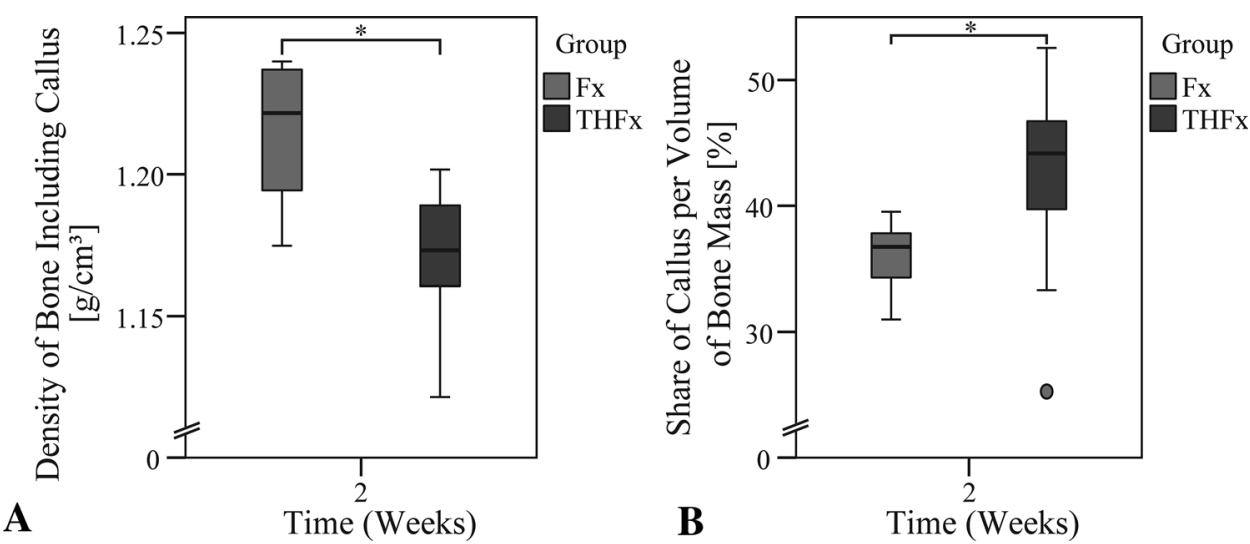

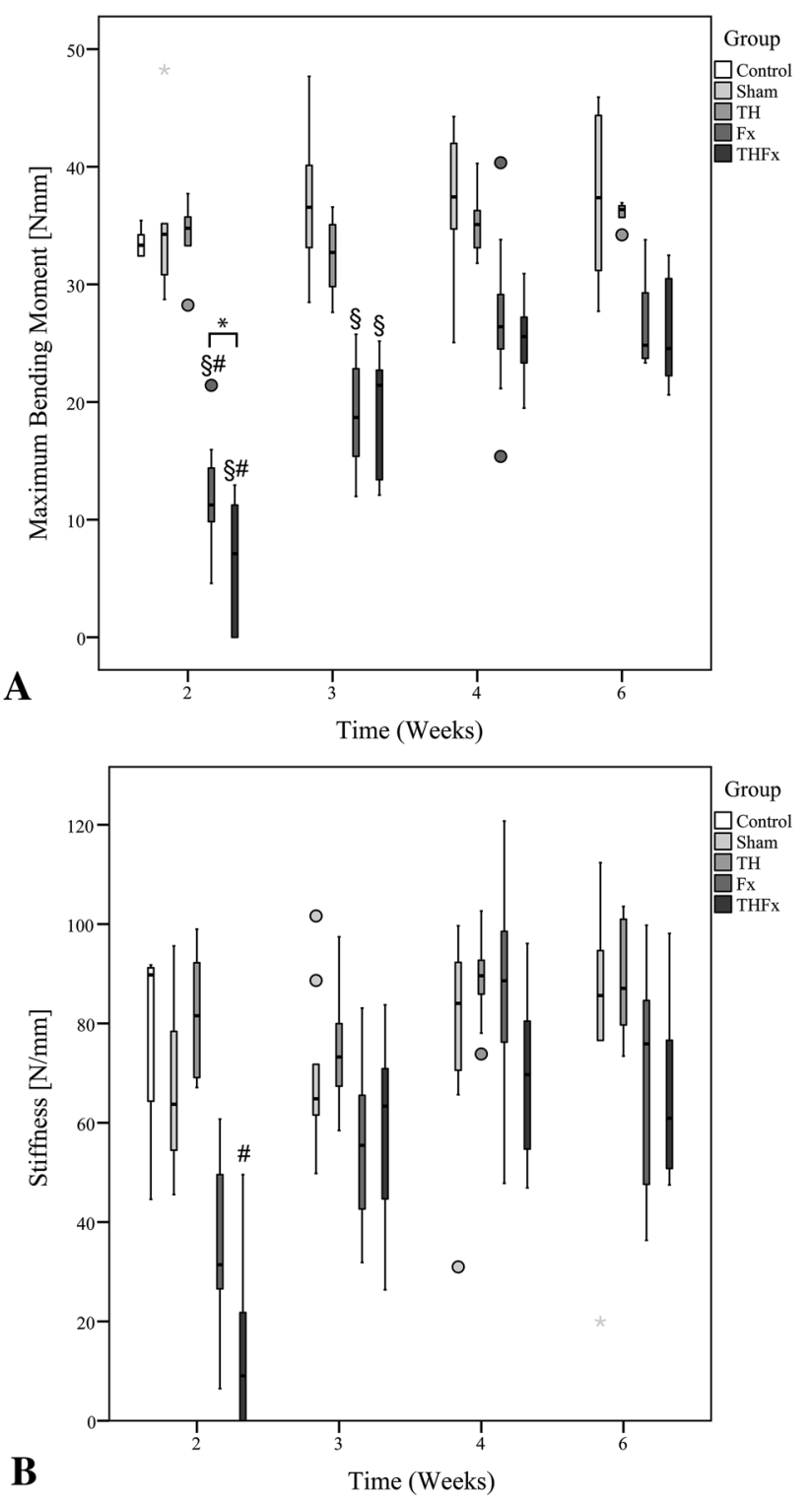

Fig. 6A-B (A) After 2 weeks the maximum bending moment was decreased in the THFx group compared with the Fx group. A difference also was seen between the THFx and Fx groups compared with the Sham and TH groups after 2 weeks. After 3 weeks, differences between the Sham group compared with the Fx and THFx groups were determined. There was no difference in maximum bending moment between the analyzed groups after 4 and 6 weeks. (B) No differences between the THFx and Fx groups could be determined at any time for stiffness; however a difference was seen between the THFx and the TH groups after 2 weeks. *p $\leq 0.05$; $\$ \mathrm{p} \leq$ 0.05 versus Sham; $\# \mathrm{p} \leq 0.05$ versus $\mathrm{TH}$.

Moreover, histologic analyses should be performed to gain more precise knowledge regarding the results determined in this study. Furthermore, one should keep in mind that the study deals with young, healthy mice whereas the effect of trauma hemorrhage in older humans with comorbidities could be different.
Starr et al. [27] conducted radiographic analyses on callus area and density and found analogous to our results no differences between Fx and THFx groups in weekly examinations up to 4 weeks after surgery in a goat model. Lichte et al. [16] performed a $\mu \mathrm{CT}$ analysis in a study setting which was similar to our model. They observed delayed fracture healing in the THFx group after 3 weeks in mice via histologic analysis, but found no differences in bone mineral density, tissue mineral density, and bone volume versus tissue volume analyzed by $\mu \mathrm{CT}$. These findings are contrary to our results of a decreased density of bone including callus in the THFx group after 2 weeks. Biomechanical analyses by Lichte et al. [16], Bumann et al. [6], and Starr et al. [27] showed no differences after biomechanical testing between $\mathrm{Fx}$ and THFx groups. Lichte et al. [16] found no difference in failure strength between the Fx and THFx groups after 3 weeks. Starr et al. [27] identified no differences in callus density, stiffness, or fracture torque 4 weeks after surgery. In contrast to our study showing a decreased maximum bending moment in the THFx group compared with the Fx group after 2 weeks in mice, Bumann et al. [6] observed no differences in higher flexural rigidity $(\mathrm{p}=0.07)$ and failure load $(\mathrm{p}=0.06)$ in the THFx group compared with the Fx group after 4 weeks in rats.

The different results could be explained by various study designs and time of analysis. In addition, the models of hemorrhagic shock were different. In the studies of Bumann et al. [6] and Starr et al. [27] a volume-controlled trauma hemorrhage was chosen. Bumann et al. [6] performed hemorrhagic shock of $12 \mathrm{~mL} / \mathrm{kg}(17 \%-22 \%$ of the whole blood) or until a mean arterial blood pressure of 60 $\mathrm{mm} \mathrm{Hg}$ was reached. This was maintained for 20 minutes followed by isovolumetric reperfusion with $6 \%$ hydroxyethyl starch. Starr et al. [27] collected 30\% of the whole blood in 15 minutes and maintained the shock for 30 minutes. Reperfusion consisted of two times the shed blood volume during 30 minutes with lactated Ringer's solution. Our model and that of Lichte et al. [16] consisted of a pressure-controlled hemorrhagic shock with a mean arterial blood pressure of $35 \pm 5 \mathrm{~mm} \mathrm{Hg}$ (30\%-40\% in our study), maintained for 90 minutes. In our study the shock was followed by reperfusion with four times the shed blood volume for 30 minutes, whereas Lichte et al. [16] infused only two times the shed blood volume with Ringer's solution. The advantage of the pressure-controlled hemorrhagic shock model is that it is standardized and reproducible in contrast to the volume-controlled model [22]. Moreover, all studies performed fracture by bending of different bones of different species. The major difference in the study by Starr et al. [27] is the use of a large animal model unlike the small animal models in all other studies mentioned here. Apart from that, Bumann et al. [6] 
and Starr et al. [27] used tibias of rats or goats, whereas Lichte et al. [16] used femurs of mice, as in our study. Moreover, Bumann et al. [6] and Lichte et al. [16] stabilized the fracture by a kind of intramedullary fixation in contrast to our study and the study of Starr et al. [27] where external fixation was used.

Our decision to use external fixation of the osteotomy follows the current scientific knowledge of the Damage Control Orthopedics concept, which is the standard treatment procedure for severely injured patients in our trauma department [13]. The Damage Control Orthopedics concept postpones definitive surgical stabilization of the fracture in severely injured patients to a later time when the acute phase of systemic recovery is over and an external fixator is used to create temporary fracture stability [13]. By contrast, the Early Total Care concept stands for definitive fracture management during the early phase of multiple trauma, performing extended surgical procedures like implanting intramedullary nails [13]. Especially severely injured patients benefit from the Damage Control Orthopedics concept in contrast to the Early Total Care concept which more often leads to pulmonary complications, a higher inflammatory response, and increases the incidence of organ dysfunction in patients with multiple trauma [13].

Based on our in vivo mouse model, we conclude that trauma hemorrhage has an influence during the early course of the fracture-healing process but not at later times that were assessed in our study. In a future study, the effect of reperfusion with whole blood in contrast to Ringer's solution, and the effect of exposure to $100 \%$ oxygen after hemorrhagic shock will be analyzed. These treatments might diminish the oxygen deficiency caused by reduced blood circulation throughout the hemorrhagic shock and therefore prevent delayed fracture healing. To provide insights in the underlying mechanisms of delayed fracture healing, histologic and signaling pathways analyses have to be included in future studies using this model, which hopefully will uncover promising mechanisms to enhance fracture healing in patients with trauma hemorrhage. Apart from that, it could be beneficial to analyze if the use of an intramedullary pin instead of an external fixator would lead to different results.

Acknowledgments We thank Claudia Pütz (Trauma Department, Hannover Medical School, Hannover, Germany) for her skillful assistance in laboratory work and Anna Katharina Marten (Small Animal Clinic, University of Veterinary Medicine, Hannover, Germany) for performing the $\mu \mathrm{CT}$ scans.

\section{References}

1. American Society of Agricultural and Biological Engineers. Standard: ASABE S459: Shear and Three-Point Bending Test of
Animal Bone. Available at: http://standards.globalspec.com/std/ 10074148/asabe-s459. Accessed June 20, 2017.

2. Andermahr J, Elsner A, Brings AE, Hensler T, Gerbershagen H, Jubel A. Reduced collagen degradation in polytraumas with traumatic brain injury causes enhanced osteogenesis. J Neurotrauma. 2006;23:708-720.

3. Barkhausen T, Probst C, Hildebrand F, Pape HC, Krettek C, van Griensven M. Insulin therapy induces changes in the inflammatory response in a murine 2-hit model. Injury. 2009;40:806-814.

4. Beil FT, Barvencik F, Gebauer M, Seitz S, Rueger JM, Ignatius A, Pogoda P, Schinke T, Amling M. Effects of estrogen on fracture healing in mice. J Trauma. 2010;69:1259-1265.

5. Bhandari M, Tornetta P 3rd, Sprague S, Najibi S, Petrisor B, Griffith L, Guyatt GH. Predictors of reoperation following operative management of fractures of the tibial shaft. J Orthop Trauma. 2003;17:353-361.

6. Bumann M, Henke T, Gerngross H, Claes L, Augat P. Influence of haemorrhagic shock on fracture healing. Langenbecks Arch Surg. 2003;388:331-338.

7. Choudhry MA, Chaudry IH. 17beta-Estradiol: a novel hormone for improving immune and cardiovascular responses following trauma-hemorrhage. J Leukoc Biol. 2008;83:518-522.

8. Dumont C, Kauer F, Bohr S, Schmidtmann U, Knopp W, Engelhardt T, Sturmer EK, Sturmer KM. Long-term effects of saw osteotomy versus random fracturing on bone healing and remodeling in a sheep tibia model. J Orthop Res. 2009;27:680686.

9. European Commission. Legislation for the protection of animals used for scientific purposes. Directive 2010/63/EU. Available at: http://ec.europa.eu/environment/chemicals/lab_animals/legislation_ en.htm. Accessed June 20, 2017.

10. Goldberg VM, Powell A, Shaffer JW, Zika J, Bos GD, Heiple KG. Bone grafting: role of histocompatibility in transplantation. $J$ Orthop Res. 1985;3:389-411.

11. Hak DJ, Fitzpatrick D, Bishop JA, Marsh JL, Tilp S, Schnettler R, Simpson H, Alt V. Delayed union and nonunions: epidemiology, clinical issues, and financial aspects. Injury. 2014;45(suppl 2):S3S7.

12. Harkness JE, Wagner JE. Biology and husbandry. The Biology and Medicine of Rabbits and Rodents. 3rd Ed. Philadelphia, PA: Lea \& Febiger. 1989; 372-373.

13. Hildebrand F, Giannoudis P, Krettek C, Pape HC. Damage control: extremities. Injury. 2004;35:678-689.

14. Kleber C, Becker CA, Malysch T, Reinhold JM, Tsitsilonis S, Duda GN, Schmidt-Bleek K, Schaser KD. Temporal profile of inflammatory response to fracture and hemorrhagic shock: proposal of a novel long-term survival murine multiple trauma model. J Orthop Res. 2015;33:965-970.

15. Kuner EH, Berwarth H, Lucke SV. [Treatment principles in aseptic pseudarthrosis][in German]. Orthopade. 1996;25:394404.

16. Lichte P, Kobbe P, Pfeifer R, Campbell GC, Beckmann R, Tohidnezhad M, Bergmann C, Kadyrov M, Fischer H, Gluer CC, Hildebrand F, Pape HC, Pufe T. Impaired fracture healing after hemorrhagic shock. Mediators Inflamm. 2015;2015:132451.

17. Lucas TS, Bab IA, Lian JB, Stein GS, Jazrawi L, Majeska RJ, Attar-Namdar M, Einhorn TA. Stimulation of systemic bone formation induced by experimental blood loss. Clin Orthop Relat Res. 1997;340:267-275.

18. Meybohm P, Zacharowski K. Hämorrhagischer schock. In Marx G, Muhl E, Zacharowski K, eds. Die Intensivmedizin. Berlin, Germany: Springer; 2015:1-23.

19. Mitruka BM, Rawnsley HM. Clinical, Biochemical and Haematological Reference Values in Normal Experimental Animals and Normal Humans. New York, NY: Masson Publishing; 1981. 
20. Neunaber C, Yesilkaya P, Putz C, Krettek C, Hildebrand F. Differentiation of osteoprogenitor cells is affected by traumahaemorrhage. Injury. 2013;44:1279-1284.

21. Pfeifer R, Kobbe P, Darwiche SS, Billiar TR, Pape HC. Role of hemorrhage in the induction of systemic inflammation and remote organ damage: analysis of combined pseudo-fracture and hemorrhagic shock. J Orthop Res. 2011;29:270-274.

22. Pfeifer R, Lichte P, Schreiber H, Sellei RM, Dienstknecht T, Sadeghi C, Pape HC, Kobbe P. Models of hemorrhagic shock: differences in the physiological and inflammatory response. $C y$ tokine. 2013;61:585-590.

23. Raschke MJ, Stange R. Femurschaft: Fehlstellungen, Pseudarthrosen und Infektionen. In Haas NP, Krettek C, eds. Tscherne Unfallchirurgie: Hüfte und Oberschenkel. Berlin, Germany: Springer-Verlag; 2012: 335-358.
24. Schmit-Neuerburg KP, Joka T. Principles of treatment and indications for surgery in severe multiple trauma. Acta Chir Belg. 1985;85:239-249.

25. Schneider CP, Schwacha MG, Chaudry IH. Impact of sex and age on bone marrow immune responses in a murine model of traumahemorrhage. J Appl Physiol (1985). 2007;102:113-121.

26. Simpson H, Mills LA. In Vivo Models: Animal Models for Bone Healing. Experimental Research Methods in Orthopedics and Trauma. Stuttgart, Germany: Thieme; 2015: 332-338.

27. Starr AJ, Welch RD, Eastridge BJ, Pierce W, Zhang H. The effect of hemorrhagic shock in a caprine tibial fracture model. J Orthop Trauma. 2002;16:250-256.

28. Wichmann MW, Arnoczky SP, DeMaso CM, Ayala A, Chaudry IH. Depressed osteoblast activity and increased osteocyte necrosis after closed bone fracture and hemorrhagic shock. J Trauma. 1996;41:628-633. 